\title{
Study of Annealing the Damaged HPGe Detector ${ }^{*}$
}

\author{
Jianyong Zhang' ${ }^{1}$ Xiaohu Mo',2, Xiao Cai ${ }^{1}$ \\ ${ }^{1}$ Institute of High Energy Physics, CAS, Beijing, China \\ ${ }^{2}$ University of Chinese Academy of Sciences, Beijing, China \\ Email: jyzhang@mail.ihep.ac.cn,moxh@mail.ihep.ac.cn
}

How to cite this paper: Zhang, J.Y., Mo, X.H. and Cai, X. (2021) Study of Annealing the Damaged HPGe Detector. World Journal of Nuclear Science and Technology, 11, 100-108.

https://doi.org/10.4236/wjnst.2021.112007

Received: February 23, 2021

Accepted: April 9, 2021

Published: April 12, 2021

Copyright $\odot 2021$ by author(s) and Scientific Research Publishing Inc.

This work is licensed under the Creative

Commons Attribution International

License (CC BY 4.0).

http://creativecommons.org/licenses/by/4.0/

\begin{abstract}
High purity germanium detectors have important applications in many fields. Detector's performance deteriorated significantly due to radiation of neutron. The annealing of damaged HPGe detector is expounded in this monograph. The experiment results indicate that raising the temperature to $70^{\circ} \mathrm{C}$ for five days, the restoration efficiency can reach $90 \%$.
\end{abstract}

\section{Keywords}

Annealing, HPGe Detector, Restoration Efficiency

\section{Introduction}

In 1970s, the technique for the production of ultra-pure germanium with impurity reached as low as $10^{10}$ atoms $/ \mathrm{cm}^{3}$. From then on, the usage of the High Purity Germanium (HPGe) detectors becomes widely in many fields, especially in astrophysics study [1]. However, the strong cosmic radiation damages the germanium crystal and then the detection results are affected considerably [2] [3] [4]. Therefore, from the very earlier days of application of germanium detector, the radiative damage issue has triggered a series of studies.

In order to determine the beam energy precisely, a high accuracy beam energy measurement system (BEMS) [5] [6] is constructed at the north crossing point (NCP) of the BEPCII, and has been commissioning since 2011. Shown in Figure 1 is the layout schematic of the system, whose working principle can be recapitulated as follows [7] [8]: firstly, a laser source provides a laser beam, and an optics system focuses the laser beam and guides it to collide with the electron (or positron) beam in the vacuum pipe, where the Compton backscattering process happens; after that the backscattering high energy photons are detected by the HPGe detector, which is the key instrument of BEMS. The accuracy of

${ }^{\star}$ This work is supported in part by State key laboratory of particle and detection and electronics. 


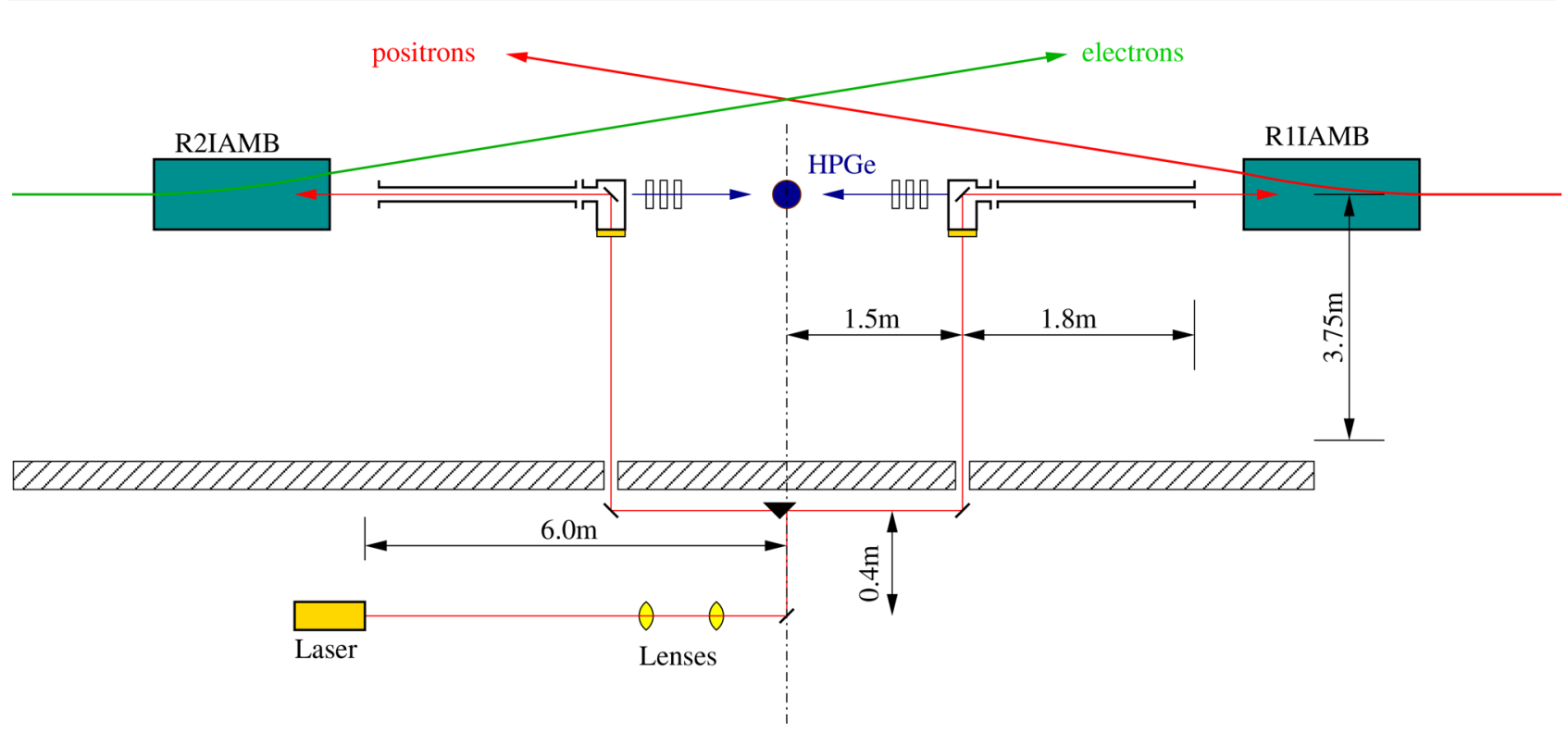

Figure 1. Simplified schematic of the beam energy measurement system. The positron and electron beams are indicated. R1IAMB and R2IAMB are accelerator magnets, and the HPGe detector is represented by the dot at the center. The shielding wall of the beam tunnel is shown cross-hatched, and the laser is located outside the tunnel.

beam energy depends solely on the detection results of HPGe detector.

Our HPGe detector locates in the storage tunnel of BEPCII, where the radiation dose is fairly high [9] [10], especially the neutrons yield serious damages on HPGe detector. The resolution of germanium detector deteriorates continuously during data taking. Such a degradation of resolution leads to the crucial deviation of beam energy measurement. Detailed studies [11] figure out that the germanium crystal was damaged heavily by neutrons. In this monograph, the annealing method is adopted to repair the resolution of the damaged HPGe detector.

\section{HPGe Detector and Resolution Restoration}

\subsection{HPGe Detector}

A P-type coaxial detector manufactured by ORTEC (model GEM25P4-70) is adopted as shown in Figure 2. The diameter and the height of the detector are $57.8 \mathrm{~mm}$ and $52.7 \mathrm{~mm}$ separately [12]. The relative efficiency is $31.2 \%$. The energy resolution for the $1.33 \mathrm{MeV}$ peak of ${ }^{60} \mathrm{Co}$ is $1.80 \mathrm{keV}$. The detector is connected to the multichannel analyzer of ORTEC DSpec Pro (MCA), which transfers data using the USB port of the computer.

Figure 3 shows the dependence of the energy resolution of HPGe detector on the data taking time. The radiation source we used here is the $1.33 \mathrm{MeV}$ peak of ${ }^{60} \mathrm{Co}$. Clearly the resolution of germanium detector deteriorates continuously.

\subsection{Radiation Damage}

The principal consequence of radiation damage by neutron is to give rise to the displacement of atoms from their lattice positions and form an interstitial defect, 


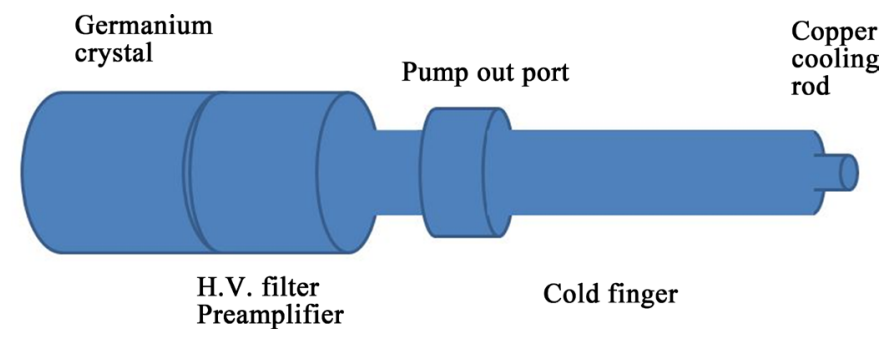

Figure 2. The HPGe detector consists of germanium crystal, preamplifier, pump out port and cold finger.

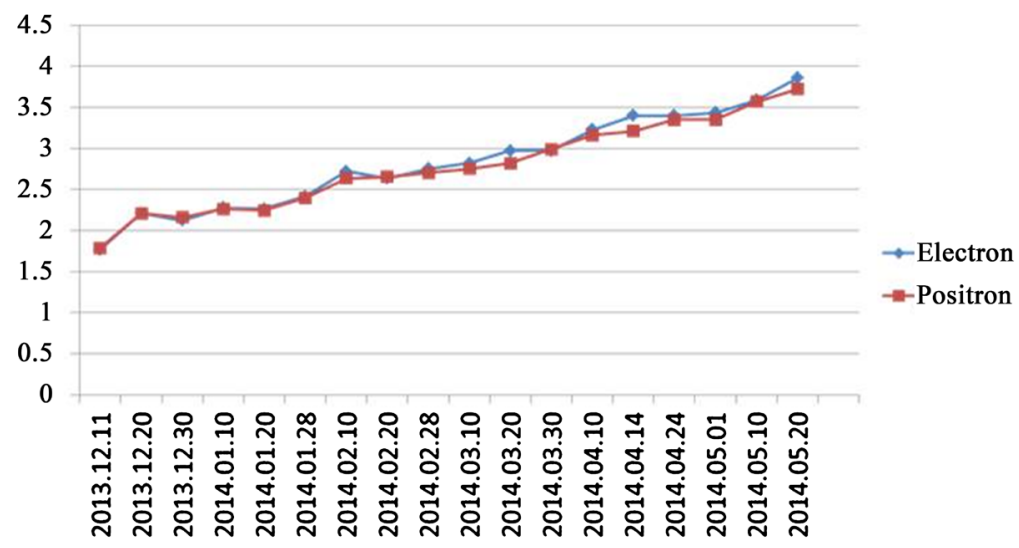

Figure 3. The dependence of resolution of HPGe detector on time. The radiation source we used here is the $1.33 \mathrm{MeV}$ peak of ${ }^{60} \mathrm{Co}$. The red square represents the detector's resolution measuring at the positron beam side, and the blue diamond represents that on the electron beam side.

some of the carriers will be trapped by these defects [13] [14]. To our experiment, the detector is used in a neutron field [15] for several months, the amount of hole trapping within the active volume of the detector is increased. In a damaged detector, the amount of charge collected is subject to a loss caused by this trapping, which will vary from pulse to pulse depending on the position of the interaction. Measured peaks in the pulse height spectrum will the show a tailing towards the low-energy side. The gradual broadening of resolution has been shown in Figure 3. Figure 4 shows the energy of resolution of HPGe detector. Before experiment the energy of resolution is $1.8 \mathrm{keV}$, while after about 6 months experiment it becomes $4.8 \mathrm{keV}$, almost about 3 fold degraded.

\subsection{Annealing Repair}

The interstitial defect is in an unstable position, and is prevented from moving back to its lattice position by an energy barrier. Raising the temperature of the crystal by quite modest amount will help the defect to its proper position and the damaged HPGe detector would be repaired.

\subsection{Annealing Methodology}

As shown in Figure 5, the procedure of annealing is as follows: the first step is to heat the crystal of the germanium detector to the temperature of $70^{\circ} \mathrm{C}$; then to 


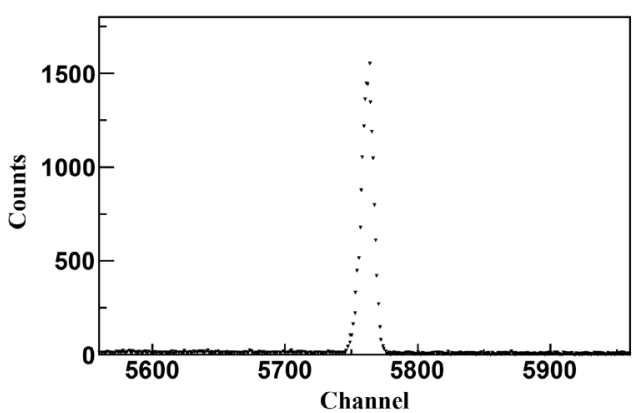

(a)

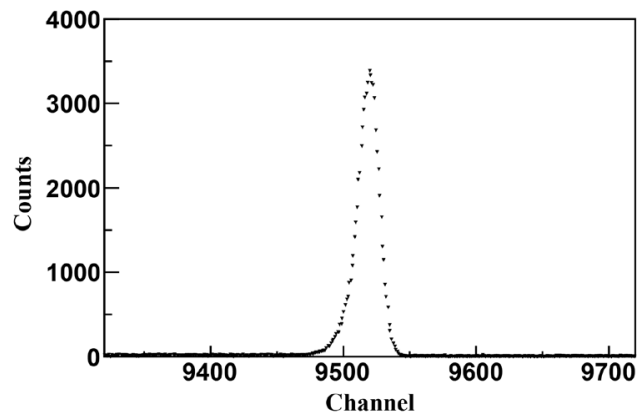

(b)

Figure 4. The resolution of HPGe detector. (a): before experiment, (b): after experiment.

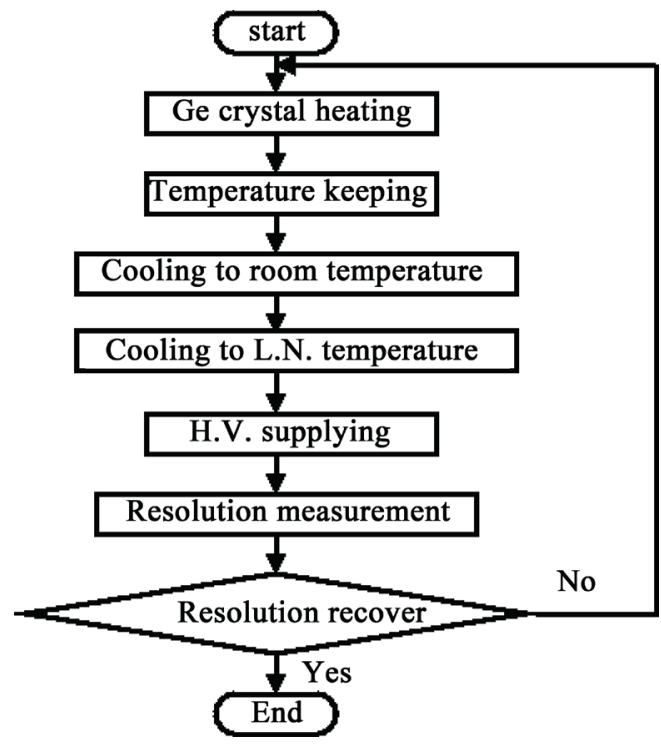

Figure 5. The flow chart of annealing experiment.

keep the temperature for five days. After that, the crystal is cooled to room temperature. The cooling system will be utilized to further decrease the temperature of the germanium crystal until about 100 Kelvin, where the HPGe detector can be worked normally. A voltage is added to bias the detector, and then measure the resolution of the detector using a special radiative source, say, ${ }^{60} \mathrm{Co}$ that is usually adopted. If the resolution is not good enough for the future usage, the annealing procedure will be iterated.

To qualitatively describe the annealing effect, the restoration-efficiency of annealing is introduced as follows [16]

$$
f_{A}=1-\frac{R-R_{0}}{R_{D}-R_{0}},
$$

where $f$ is the restoration-efficiency, the subscript $A$ indicates the annealing; $R$, $R_{0}$, and $R_{D}$ denote respectively the detector resolutions after the annealing, before experiment (without radiation damage), and after experiment (with radiation damage). According to Equation (1), if $R=R_{D}$, then $f_{A}=0$, which means there is no any restoration effect; if $R=R_{0}$, then $f_{A}=1$, which means the resolution 
is totally recovered.

\section{Annealing Technique}

\subsection{Annealing Using Stove}

At first, we use a stove to heat the germanium crystal. As shown in Figure 6, the stove model is SGL-1200Z. The dimension of stove is $74.5 \mathrm{~cm}$ long with diameter $46 \mathrm{~cm}$. The thermostat region is the center of stove range about $5 \mathrm{~cm}$, with the temperature accuracy of $\pm 1^{\circ} \mathrm{C}$. The copper cooling rod of HPGe detector is placed at the thermostat region of the stove and heated, and during heating period, a multimeter is used to monitor the temperature variation of the sensitive resister of HPGe detector. The Lab-view software is adopted to set the annealing temperature, heating velocity, and time of temperature preservation. During experiment, the annealing temperature of $120^{\circ} \mathrm{C}, 3^{\circ} \mathrm{C}$ per minute of heating velocity, and 120 hours for temperature preservation, are set. The germanium crystal is cooled down with the stove after enough preservation.

During the annealing period, the vacuum pump is used to keep the vacuum of HPGe less than 0.1 Pa. In the whole experiment, the temperature of the center region of the stove is as high as $120^{\circ} \mathrm{C}$, however, that of germanium crystal is merely $37^{\circ} \mathrm{C}$. Even the heating time increases, the temperature of the crystal remains same. This fact implies that it is difficult to enhance the temperature of germanium crystal significantly using such a radiative heating method (Table 1).

\subsection{Annealing Using Heating Belt}

Another approach, namely annealing using heating belt, is applied. Compared

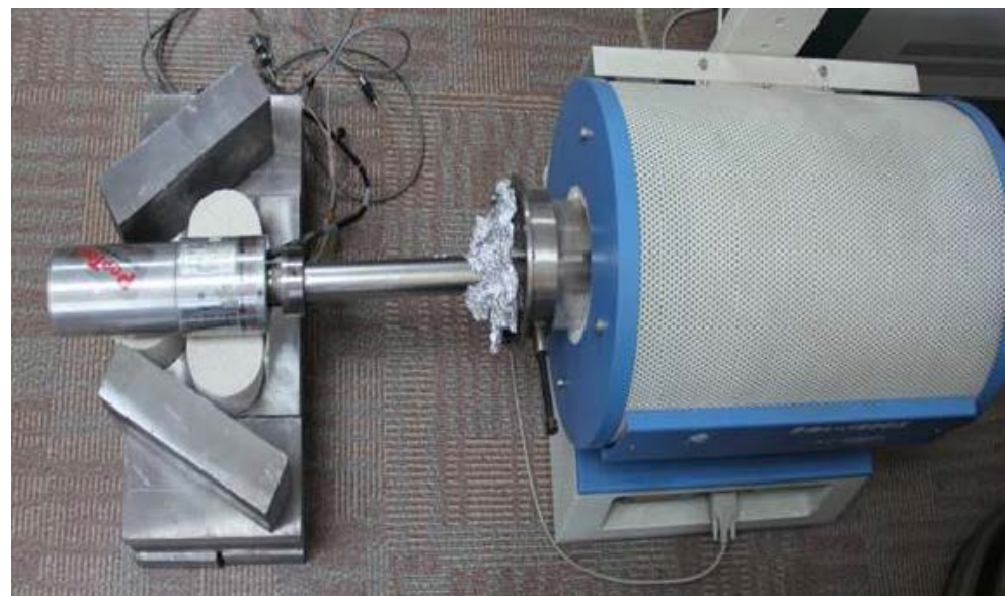

Figure 6. Annealing using stove.

Table 1. HPGe annealing with stove.

\begin{tabular}{ccccc}
\hline Time & FWHM & FW[1/5]M & $f_{A} \%$ & Remark \\
\hline 20140303 & 2.00 & 3.07 & & Before annealing \\
20140318 & 1.99 & 3.05 & 5 & After annealing \\
\hline
\end{tabular}


with the stove annealing, the heating blet uses direct contact, the heat transfer efficiency is higher.

\subsubsection{Annealing Using Heating Belt Directly}

To heat the germanium crystal, the cooling rod is enclosed by the glass fiber heating belt manufactured by Shanghai Huo Long company, with the dimension of $2 \mathrm{~cm}$ width and $50 \mathrm{~cm}$ length. Its maximum heating temperature is $250^{\circ} \mathrm{C}$. During the heating, the temperature-controlled power, made by Shanghai San Jing company is used, which has a platinum-made electric couple. Such a electric couple is inserted in the heating belt to control the working status of the heating belt. When the heating temperature exceeds the preset value, the power will shut down; while when the temperature is lower than the preset value, the power will automatically start to work again.

With the suitable vacuum, when the temperature of heating belt reaches $120^{\circ} \mathrm{C}$, that of Germanium crystal is around $44^{\circ} \mathrm{C}$, which is obviously better than the situation of the stove heating. Notice the distance between the heating point and the crystal is about $80 \mathrm{~cm}$, the quartz capsule and insulated foam are wrapped up the cooling rod to prevent from seriously heat dissipates. With such a protection, the temperature of the germanium crystal can reach $48.5^{\circ} \mathrm{C}$. The setup of heating experiment is displayed in Figure 7.

\subsubsection{Annealing Using Copper Heating Block}

The most part of the cooling rod of the germanium detector is encapsulated as a vacuum tube made by stannum alloy. The nude copper cooling rod is only 3.8 $\mathrm{cm}$ long with diameter $2.5 \mathrm{~cm}$, which is hard to fix and enclose by heating belt. Therefore, the heating efficiency is too limited.

To increase heating efficiency, a special copper ingot is designed. It is the cube with side length $6 \mathrm{~cm}$. A hole with length of $3.8 \mathrm{~cm}$ and diameter of $2.5 \mathrm{~cm}$ is drilled at the center of copper cube. At the other side of cube, deviated from the drilled hole, another hole with length of $2 \mathrm{~cm}$ and diameter of $0.5 \mathrm{~cm}$ is drilled, which is used for inserting the platinum-made electric couple.

The copper cooling rod with thermal conductive grease is put in the hole of the copper cube, then is enclosed by heating belt. The temperature-controlled power responds for the heating process. In order to heat the cube homogeneously,

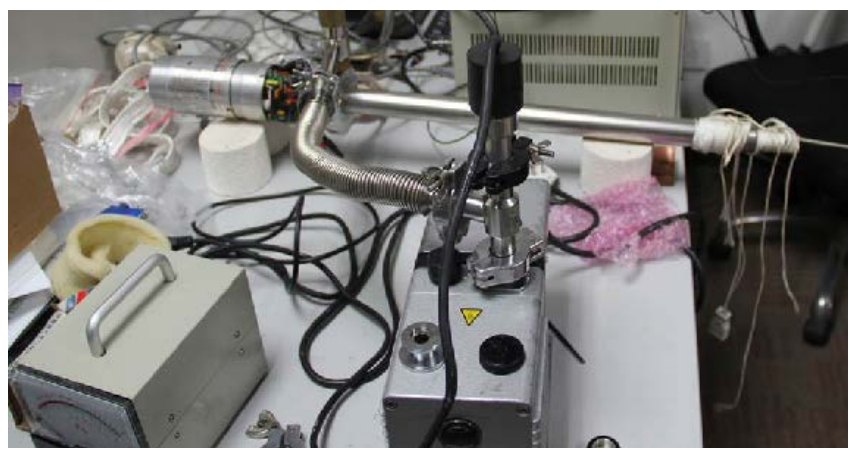

Figure 7. The copper rod of the HPGe cold finger is annealing using heating belt. 
aluminium foil is used the enclose the heating belt. In addition, the whole cooling rod is enclose with asbestos tapes to prevent heat dissipating, as shown in Figure 8.

In order to increase the crystal temperature further, two temperature-controlled heating systems are adopted. One is used to heat the copper cube, the other to heat the cooling rod. When the preset temperatures are $240{ }^{\circ} \mathrm{C}$ for the former, and $120^{\circ} \mathrm{C}$ for the latter, the temperature of germanium crystal can reach $65^{\circ} \mathrm{C}$.

\subsubsection{Optimization of Heating Block}

Improvements are made for the design of copper ingots. The sharp edges of the copper cube is unsuitable for the contact between it and the heating belt. Then a two half cylindrical copper ingots are designed, with the diameter $2.5 \mathrm{~cm}$ of center hole. The silver is used to welded the handle of the cylinder, and screws are used to control the contact between cooling rod and copper cylinder, which settle the contact issue fairly well. However, the handle of the cylinder is a burden for heating belt. As an improvement design, the internally installed-screw cylinder is manufactured as shown in Figure 9. Adopted such a cylinder for crystal heating, the temperature can reach $70^{\circ} \mathrm{C}$.

\subsection{Results}

Contact-heating method is an effective way we figured out to increase the heating

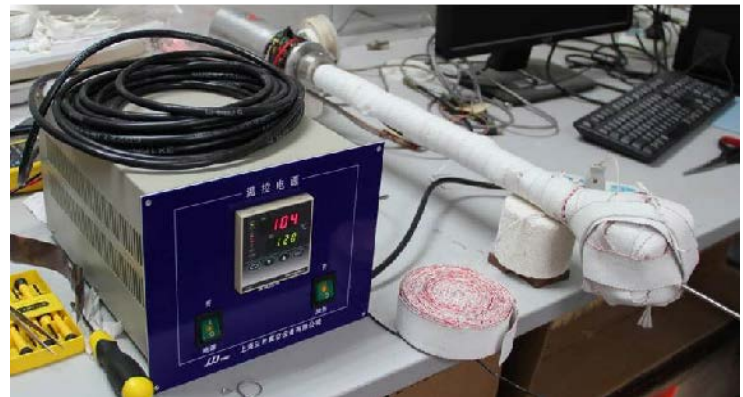

Figure 8. Annealing the copper rod of the HPGe using the heating ingot, asbestos belt is added to prevent the heat loss.

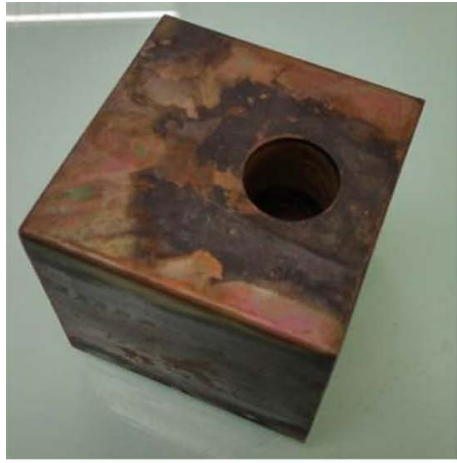

(a)

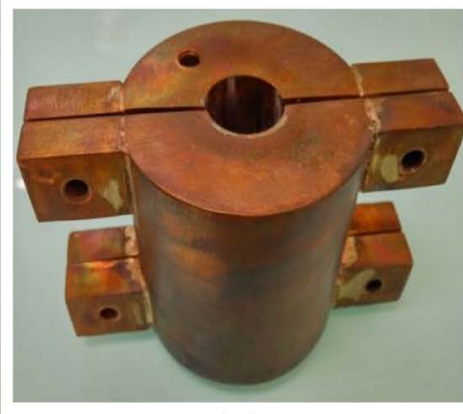

(b)

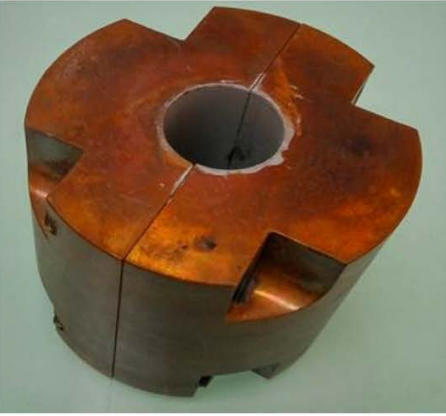

(b)

Figure 9. Optimization of the copper heating block. (a) cubic heating block, (b) columnar heating block with handles, (c) columnar heating block without handles. 


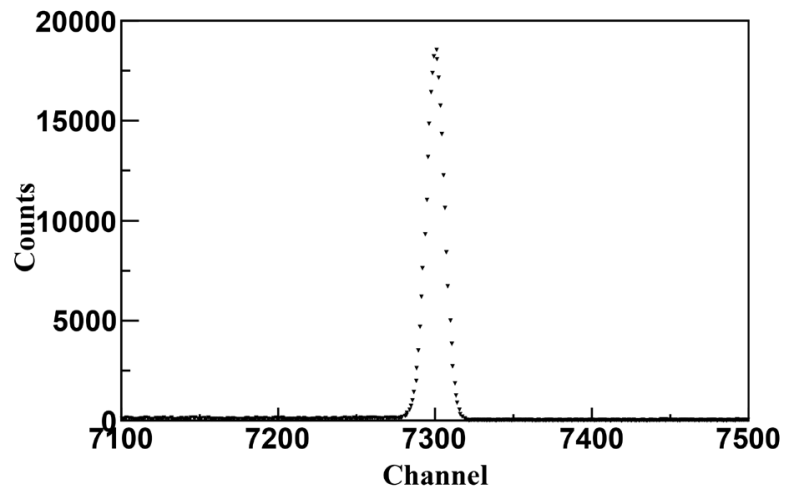

Figure 10. The energy resolution of HPGe detector of $2.5 \mathrm{keV}$ at $1.33 \mathrm{MeV}$ line of ${ }^{60} \mathrm{Co}$.

Table 2. HPGe annealing with heating belt.

\begin{tabular}{ccccc}
\hline Time & FWHM & FW[1/5]M & Annealing time (day) & $f_{A} \%$ \\
\hline 20170630 & 10.61 & 14.25 & & \\
20170716 & 7.38 & 10.28 & 1 & 36.7 \\
20170724 & 5.73 & 8.27 & 2 & 55.4 \\
20170901 & 3.90 & 5.93 & 3.5 & 76.2 \\
20180123 & 2.50 & 3.81 & 5 & 92.1 \\
\hline
\end{tabular}

temperature of crystal. It should be stress, during the heating process, the high vacuum status is of great importance for heating transform and temperature keeping. The temperature of germanium crystal can be annealing at $70^{\circ} \mathrm{C}$.

We arranged a set of experiments for annealing effect varies with annealing time as list in Table 2. It is clear that the energy resolution of the HPGe detector becomes better as the annealing time increases. Keeping the crystal temperature at $70^{\circ} \mathrm{C}$ for 5 days, the damaged HPGe detector whose resolution is $10.61 \mathrm{keV}$, can be restored to $2.5 \mathrm{keV}$ at $1.33 \mathrm{MeV}$ line of ${ }^{60} \mathrm{Co}$ (Figure 10).

\section{Summary}

Many efforts are made to restore the resolution of the damaged germanium crystal by means of annealing method. A special copper ingot is designed for cooling rod heating, the heating temperature of crystal at $70^{\circ} \mathrm{C}$ is obtained by double-temperature-controlled heating systems. The final restoration-efficiency can reach $92.1 \%$.

\section{Conflicts of Interest}

The authors declare no conflicts of interest regarding the publication of this paper.

\section{References}

[1] Haller, E.E. (1982) Detector Materials: Germanium and Silicon. IEEE Transactions on Nuclear Science, 29, 1109-1118. https://doi.org/10.1109/TNS.1982.4336330 
[2] Raudorf, T.W., et al. (1984) Performance of Reverse Electrode HPGE Coaxial Detectors after Light Damage by Fast Neutrons. IEEE Transactions on Nuclear Science, 31, 253-257.

[3] Koenen, M., et al. (1995) Radiation Damage in Large-Volume n- and p-Type High-Purity Germanium Detectors Irradiated by $1.5 \mathrm{GeV}$ Protons. IEEE Transactions on Nuclear Science, 42, 653-658.

[4] Sajo-Bohus, L., et al. (2011) HPGe Detectors Long Time Behaviour in High-Resolution $\gamma$ Spectrometry. Nuclear Instruments and Methods in Physics Research Section A: Accelerators, Spectrometers, Detectors and Associated Equipment, 648, 132-138. https://doi.org/10.1016/j.nima.2011.03.031

[5] Mo, X.-H. (2007) Study of High Precision $\tau$ Mass Measurement at BESIII. Nuclear Physics B-Proceedings Supplements, 169, 132-139.

https://doi.org/10.1016/j.nuclphysbps.2007.02.107

[6] Abakumova, E.V., et al. (2011) The Beam Energy Measurement System for the Beijing Electron-Positron Collider. Nuclear Instruments and Methods A, 659, 21-29.

[7] Mo, X.-H., et al. (2008) Working Principles of the Energy Measurement System at BEPCII. Chinese Physics C, 32, 995. https://doi.org/10.1088/1674-1137/32/12/011

[8] Zhang, J. (2017) Beam Energy Measurement System for BEPCII. JINST, 12, C07019. https://doi.org/10.1088/1748-0221/12/07/C07019

[9] Mo, X.-H., Zhang, J.-Y., Zhang, Q.-J., et al. (2011) Study of Radiation Background at the North Crossing Point of the BEPCII in Collision Mode. CPC (HEP NP), 35 , 642-655.

[10] Mo, X.-H., Zhang, J.-Y. and Zhang, T.-B. (2009) Measurement of Radiation Dose at the North Interaction Point of BEPC II. CPC (HEP NP), 33, 914-921. https://doi.org/10.1088/1674-1137/33/10/017

[11] Zhang, J.Y., Cai, X., Mo, X.H., et al. (2019) Energy Deviation Study of BEMS at BEPCII. Nuclear Physics B, 939, 391-404. https://doi.org/10.1016/j.nuclphysb.2018.12.023

[12] Solid-State Photon Detector Operators Manual, GEM Series-HPGe Coaxial Detector System, ORTEC.

[13] Knoll, G.F. (1979) Radiation of Detection and Measurement. John Wiley \& Sons, New York.

[14] Gilmore, G.R. (2008) Practical Gamma Ray Spectrometry and Edition. John Wiley and Sons, Ltd., New York. https://doi.org/10.1002/9780470861981

[15] Zhang, C. and Ma, L. (2015) Design and Development of Accelerator for Beijing Electron and Positron Collider Major Reconstruction Project. Shanghai Science and Technology Press, Shanghai. (In Chinese)

[16] Zhang, J.Y., Mo, X.H. and Cai, X. (2020) Annealing Restoration of HPGe Detector. Radiation Detection Technology and Methods, 4, 106-109.

https://doi.org/10.1007/s41605-019-00155-y 